\title{
An Empirical Study on Structural, Optical and Electronic Properties of $\mathrm{ZnO}$ Nanoparticles
}

\author{
Shyam Sundar Pareek ${ }^{1}$ Kapil Pareek ${ }^{2}$ \\ ${ }^{I}$ (Department of Physics, IASE Deemed University, GVM, Sardarshar,Churu (Rajasthan), India) \\ ${ }^{2}$ (Research scholar, Department of Chemistry, National University of Singapore, Singapore)
}

\begin{abstract}
In this work, the synthesis routes for zinc oxide $(\mathrm{ZnO})$ nanoparticles and optical \& electronic properties of $\mathrm{ZnO}$ nanoparticles have been demonstrated. $\mathrm{ZnO}$ has many potential applications including sunscreens, biosensors, food additives, pigments, rubber manufacture, and electronic materials. Because of the unique quantum confinement effects of $\mathrm{ZnO}$ nanoparticles, synthesis and characterization of $\mathrm{ZnO}$ nanoparticles has been received a great attention in contemporary materials science. The synthesized $\mathrm{ZnO}$ materials can be further characterized by many characterization techniques in order to confirm synthesis. In this case, X-ray diffraction (XRD is a very powerful tool to analyse the structure of the material. Surface characterization such as Scanning Electron Microscopy (SEM) and Transmission electron microscopy (TEM)could be very useful to understand the morphology of the nanoparticles. Also, Fourier transform infrared spectroscopy (FTIR) is helpful to identify particular chemical bonding in the synthesized materials. Thermal stability and thermal transition can be measured by thermal gravimetric analysis (TGA) and differential scanning calorimetry (DSC), respectively. The optical properties of ZnO nanoparticles can be measured by UV-VIS spectroscopy.
\end{abstract}

Key Words: Nanoparticle, Optical and Electronic Property, Thermal analysis, $X$-ray diffraction.

\section{Introduction}

The world is surmounting on the roof of science and technology where materials such as semiconductor has tremendous impact on the economy. In this case, Germanium $(\mathrm{Ge})$ and $\operatorname{Silicon}(\mathrm{Si})$ are famous example semiconductor materials. Germanium gets famous due to possession of property like low melting point and lack of natural occurring germanium oxide to prevent the surface from electrical leakage where as silicon dominates the commercial market for its better fabrication technology and application to integrated circuits for different purposes. As time passes on, the rapid growing world demands speed along with technology. This need was very well fulfilled by GaAs which easy the path for the design of high speed and optoelectronic devices. GaAs which is a direct band gap semiconductor possessing higher carrier mobility and higher effective carrier velocity in comparison to $\mathrm{Si}$ makes it better suited for optoelectronics devices. But this do not leads to the completion of requirement for the future world, something more is required that is high temperature electronics devices.

The world therefore now demands a material that should possess inherent properties like larger band gap, higher electron mobility as well as higher breakdown field strength. So on making investigation about such a material the name of compound comes out is "Zinc Oxide" which is a wide gap semiconductor material very well satisfying the above required properties. Not only has this Zinc oxide possessed many versatile properties for UV electronics, spintronic devices and sensor applications. Also $\mathrm{ZnO}$ has been commonly used in its polycrystalline form over hundred years in a wide range of applications. This ignites many research minds all over the world and creates enthusiasm to develop proper growth and processing techniques for the synthesis of Zinc oxide. Zinc oxide is also known as "Lu-Gan-Stone" in China, Zinc oxide has been used in medical treatment for quite number of years in China. The research on $\mathrm{ZnO}$ is catching fire right from the beginning of 1950 , with a number of reviews on electrical and optical properties like $\mathrm{N}$-type conductivity, absorption spectra and electroluminescence decay parameter.

The nature of excitonic molecule in semiconductors which was discussed by Haynes aiding a key publication by Park concerned with excitonic emission of $\mathrm{ZnO}$ is the very first footstep put for the growth and development of $\mathrm{ZnO}$ research in the mid of 1960. In 1966 the Raman Effect study of ZnO by Damen and Porto leads to the identification of $\mathrm{ZnO}$ phonon energies. The decade of 1970 for $\mathrm{ZnO}$ passes away in manufacturing of simpler $\mathrm{ZnO}$ devices like ceramic varistors, piezoelectric transducers etc. also with a progress in the study of variety of characterization techniques such as cathodluminescence(CL), capacitance - voltage studies(CV), electrical conduction and so on. When the researchers got fade off continues work on characterization technique for a decade, search for different growth procedure and techniques begin.In 1980 a lot of work on high quality thin films of $\mathrm{ZnO}$, metallo-organic chemical vapour deposition (MOCVD), spray pyrolysis and radio frequency magnetron sputtering (RF) has been done extensively. Overall much of the research in 1980's attempted to resolve the issues concerning with lack of satisfactory material for device fabrication. But finally at the end of 
the decade there were no significant increment in the number of papers. The twilight of stars sprinkles and a new era for research of $\mathrm{ZnO}$ begins actually in 1990 with a uniform growth in number of publications related to both characterization and growth techniques. In 1990's, newer growth methods were used including pulsed laser deposition (PLD) and molecular beam epitaxy (MBE). At the end of late 1990 large scale commercial ZnO comes to the picture.

In conclusion, Zinc oxide $(\mathrm{ZnO})$ is a wide bandgap $(3.37 \mathrm{eV})$ semi-conductor having a high electronhole binding energy $(60 \mathrm{meV})$ and important applications in electronics, optics, optoelectronics, laser and lightemitting diode. The piezoelectric and pyroelectric properties of $\mathrm{ZnO}$ make it a great candidate for sensors, transducers, ener-gy generators and photocatalysis for hydrogen produc-tion. $\mathrm{ZnO}$ is also a green material that is bio-compatible, biodegradable and bio-safe for medical applications and environmental science.

\section{Objective of Study}

In this study, the numerous methods for syntheses of $\mathrm{ZnO}$ nanoparticles are discussed. The detailed characterization techniques to characterize the synthesized nanoparticles are explained in the subsequent sections.

\subsection{Methods for synthesis of Nanomaterials}

The synthesis of nanomaterial can be well accomplished by two approaches. Firstly, by "Bottom Up" method where small building blocks are produced and assembled into larger structures. Here the main controlling parameters are morphology, crystallinity, particle size, and chemical composition. Examples: chemical synthesis, laser trapping, self-assembly, colloidal aggregation, etc and secondly, by "Top Down" method where large objects are modified to give smaller features. For example: film deposition and growth, nano imprint /lithography, etching technology, mechanical polishing etc. the main reason of alteration in different mechanical, thermal and other property is due to increase in surface to volume ratio. Synthesis of nanomaterial is most commonly done based on three strategies i.e.

1. Liquid-phase synthesis.

2. Gas-phase synthesis.

3. Vapour-phase synthesis.

\subsubsection{Liquid-Phase Synthesis}

Under liquid phase synthesis the techniques used for synthesis are:

a. Co-precipitation.

b. Sol-gel Processing.

c. Micro-emulsions.

d. Hydrothermal/Solvo-thermal Synthesis.

e. Microwave Synthesis.

f. Sono-chemical Synthesis.

g. Template Synthesis.

\subsubsection{Gas-Phase Synthesis}

Super saturation achieved by vapourizing material into a background gas, then cooling the gas.

\subsubsection{Vapour-phase synthesis.}

a. Inert Gas Condensation

b. Pulsed Laser Ablation

c. Spark Discharge Generation

d. Ion Sputtering

e. Chemical Vapour Synthesis

f. Spray Pyrolysis

g. Laser Pyrolysis/ Photochemical Synthesis

h. Thermal Plasma Synthesis

i. Flame Synthesis

j. Flame Spray Pyrolysis

k. Low-Temperature Reactive Synthesis

Nanostructured materials can have significantly different properties, depending on the chosen fabrication route. Each method offers some advantages over other techniques while suffering limitation from the others. 


\section{Basic Physical Parameter, Crystal And Surface Structure Of Zno Nanostructures}

General, Zinc oxide crystallizes in two main forms, hexagonal wurtzite and cubic zinc blende but the ( $\mathrm{B}_{4}$ type) wurtzite structure is obtained only at optimum pressure and temperature. In an ideal wurtzite crystal, with a hexagonal close-packed lattice type, has lattice parameters, $a_{0}=0.32495 \mathrm{~nm}$ and $c_{o}=0.52069 \mathrm{~nm}$, in the ratio of $\mathrm{c}_{\mathrm{o}} / \mathrm{a}_{\mathrm{o}}=1.602$, and it belongs to the space group of $P 63 \mathrm{mc}$ and is characterized by two interconnecting sub lattices of $\mathrm{Zn}^{2+}$ and $\mathrm{O}^{2-}$ where each anion is surrounded by four cations at the corners of a tetrahedron with a typical $s p 3$ covalent bonding. Among different phases of $\mathrm{ZnO}$, based on the first principle periodic Hartree-Fock linear combination of atomic orbitals theory the wurtzite is found to be the most thermodynamically stable phase. Tetrahedral symmetry plays a vital role for the polarity of $\mathrm{ZnO}$ that arises along the hexagonal axis. Piezoelectricity and spontaneous polarization are the direct consequence of polar symmetry of $\mathrm{ZnO}$ along the hexagonal axis. The structure of $\mathrm{ZnO}$, can be described as a number of alternating planes composed of tetrahedrally coordinated $\mathrm{O}^{2-}$ and $\mathrm{Zn}^{2+}$ ions, stacked alternately along the $\mathrm{C}$-axis (Fig.1.)The oppositely charged ions produce positively charged(0001)-Zn and negatively charged (0001)-O polar surfaces, resulting in a normal dipole moment and spontaneous polarization along the c-axis, as well as a divergence in surface energy .The polar faces are more stable than non polar faces. The root cause for the natural $\mathrm{N}$-type nature of $\mathrm{ZnO}$ is due to the sensitiveness of $\mathrm{ZnO}$ lattice constants to the presence of structural point defects (vacancies and interstitials) and extended defects (threading/planar dislocations) that are commonly found in $\mathrm{ZnO}$ resulting in a nonstoichiometric compound $\mathrm{Zn}_{1+\mathrm{d}} \mathrm{O}$ with an excess zinc .These excess zinc atoms have the tendency to function as donor interstitials that give its natural $\mathrm{N}$-type conductivity. In ionic form, the excess zinc exist as $\mathrm{Zn}+$ interstitials that are mobile and they tend to occupy special interstitial sites with Miller index $(1 / 3,2 / 3,0.875)$ as shown in Fig.1.These special sites offer passage routes for zinc interstitials to easily migrate within the $\mathrm{ZnO}$ wurtzite structure.

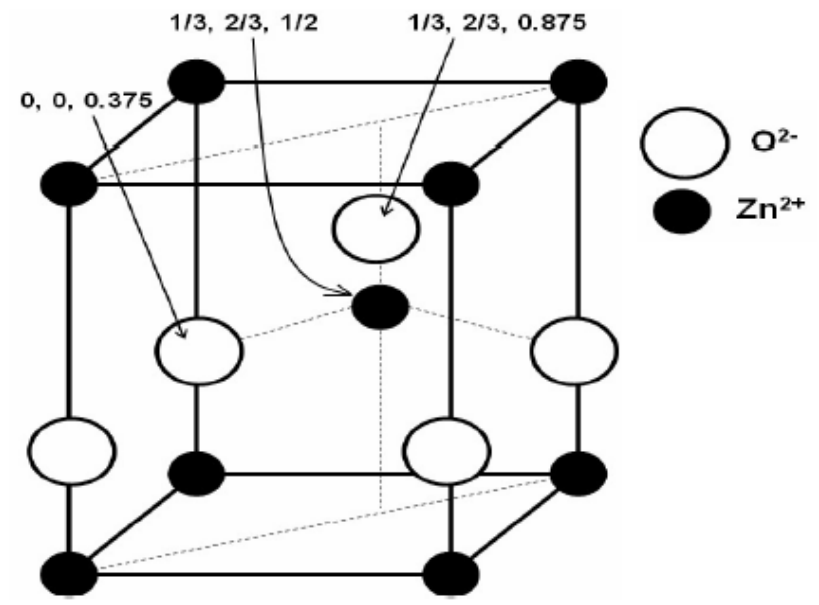

Figure 1: $\mathrm{ZnO}$ unit cell with ionic positions of zinc and oxygen atoms. Redrawn from

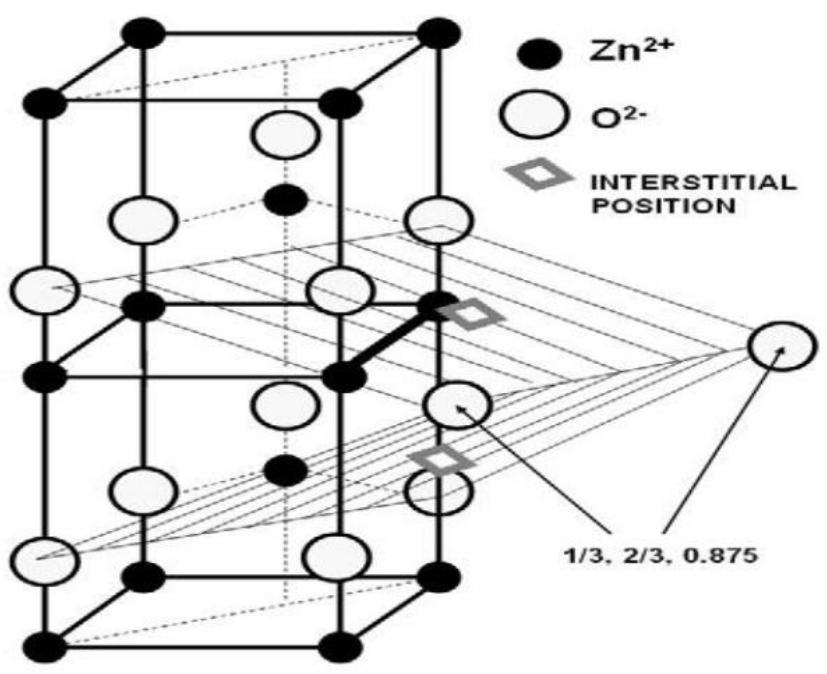

Figure 2: Zinc interstitial sites in the $\mathrm{ZnO}$ wurtzite lattice. Redrawn from 


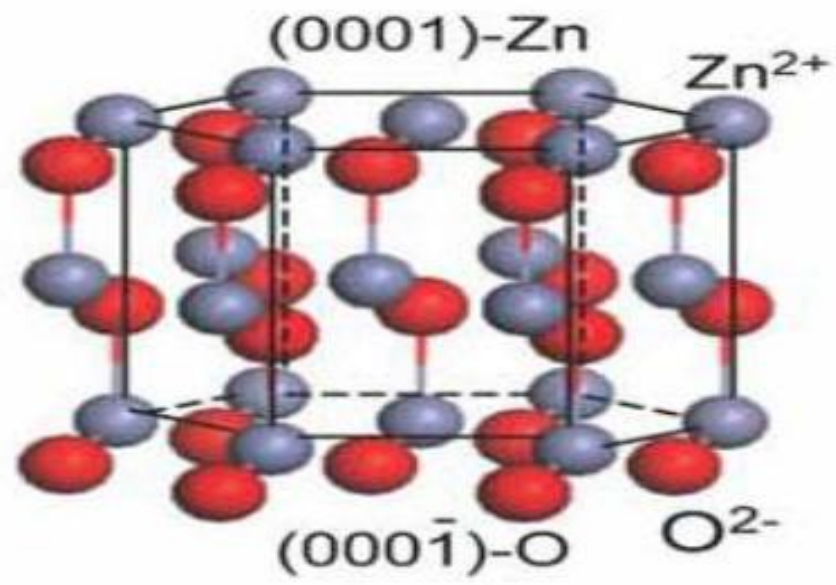

Figure 3: The wurtzite structure model of $\mathrm{ZnO}$. The tetrahedral coordination of $\mathrm{Zn}-\mathrm{O}$ is shown.

\section{Optical And Electronic Property Of Zno Nanostructures}

The most important factor responsible for a material to show a better optoelectronic property is the large exciton binding energy and this property is possessed by Zinc oxide having binding energy of 60mev which could be attended at and above room temperature due to excitonic recombination. The process of optical absorption and emission have been influenced by bound excitons which are extrinsic transition related to dopants or defects thereby usually responsible for creating discrete electronic states in the band gap. Theoretically, neutral or charged donors and acceptors are the members by which exciton could be bound with and it merely depends on the band structure of semiconductor material. Thus exciton which is a bound system it does not requires traps to localize carriers and recombines with high efficiency. The piezoelectrically induce field makes deeper exciton of $\mathrm{ZnO}$ more stable against field ionization. Magnesium doped zinc oxide possesses a wide range of sensing spectra between (200-280)nm which makes it suitable to tune for UV-B and UV-C and can be made applicable for various fields such as solar UV radiation monitoring, ultrahightemperature flame detection etc. Zinc oxide films made from single crystal shows directionally dependent optical properties due to which it can be applied for modulation of UV radiation. The current example of it is the designed model of $\mathrm{ZnO}$ modulator with a contrast of 70:1 and operation speed of 100ps. Also high breakdown stress and high saturation velocity of zinc oxide increases its demand for the different electronic application.PL spectra of $\mathrm{ZnO}$ nanowire shows increase of green emission intensity with a decrease of nanowire diameter and continuous reduction of diameter of $\mathrm{ZnO}$ nanowire gives quantum size effect and due to this size confinement exciton binding energy is enhanced. Transport characteristics and interaction of phonon with free carrier have impact on the performance of optoelectronic devices which can be acquired by the knowledge of vibrational properties of the material. The excellent emitting power of $\mathrm{ZnO}$ has been investigated through different reports and line width of excitonic recombination is as narrow as $40 \mu \mathrm{eV}$ with fine spectroscopic details have been observed. The refractive index of wurtzite $\mathrm{ZnO}$ as reported is $\mathrm{n}_{\mathrm{w}}=2.008$ and $\mathrm{n}_{\mathrm{e}}=2.029$.

\section{Energy Band Gap}

As reported from various literatures the band gap of $\mathrm{ZnO}$ films mostly depends on the carrier concentration and is found to be $3.37 \mathrm{ev}$ on basis of carrier concentration of $1018-1020 / \mathrm{cm} 3$. Anamolous change (increase) in band gap has been observed when the carrier concentration is $5 \times 1018 / \mathrm{cm} 3$, and then a sudden decline in band gap, when concentration changes to $3-4 \times 1019 / \mathrm{cm}^{3}$..Quantum confinement of electrons in small grains created by potential barriers at the grain boundaries are thought to responsible for the drastic change in band gap. Sometimes also at higher doping concentration on a blue shift towards shorter wavelength has been observed and can be explained on the basis of Burstein-Moss effect. According to which increase of the carrier concentration due to Al doping results in a shift of the Fermi level and block some of the lowest states, thereby causing widening of the band gap resulting in the blue-shift of the absorption tail. The band gap of $\mathrm{ZnO}$ as calculated by local density approximation (LDA) is found to be $3.77 \mathrm{ev}$ which mostly accounts for the $\mathrm{Zn} 3 \mathrm{~d}$ electrons as shown in the fig. 4 The $\mathrm{ZnO}$ having direct band gap is very well indicated by the valence band maxima and lowest conduction band minima both occurring at the same $\Gamma$ point of $\mathrm{k}=0$. $\mathrm{Zn} 3 \mathrm{~d}$ levels are indicated by bottom ten bands (occurring around 9ev) and $\mathrm{O} 2 \mathrm{p}$ bonding states are highlighted by next six bands from $-5 \mathrm{eV}$ to $0 \mathrm{eV}$. The empty $\mathrm{Zn} 3 \mathrm{~s}$ levels signified by first two conduction band states are mainly $\mathrm{Zn}$ localized. Crystallization of $\mathrm{ZnO}$ mostly favourable in wurtzite symmetry and crystal field splitting as well as spin orbit interaction results in three states say A, B \&C fig.4. The symmetry of the A valence sub band is considered to be 
$\Gamma_{7}$ based on the polarization properties of the free exciton transitions where as contradicting to it the most recent magneto-optical studies of the free exciton transition fine structure interpreted the symmetry to be $\Gamma_{9}$. But the controversy is continuing as before 40 years ago. It has been reported in undoped films grown by MBE a new line at $3.32 \mathrm{eV}$ was reported $(\mathrm{ZnO}$ on $\mathrm{CaF} 2(111))$ which isquite close to the $3.35 \mathrm{eV}$ line for $\mathrm{ZnO}$ on $a$-plane sapphire presented by Kato (taking into account the different strain situations in the films). Both groups assigned the recombination as caused by excitons bound to neutral acceptors.
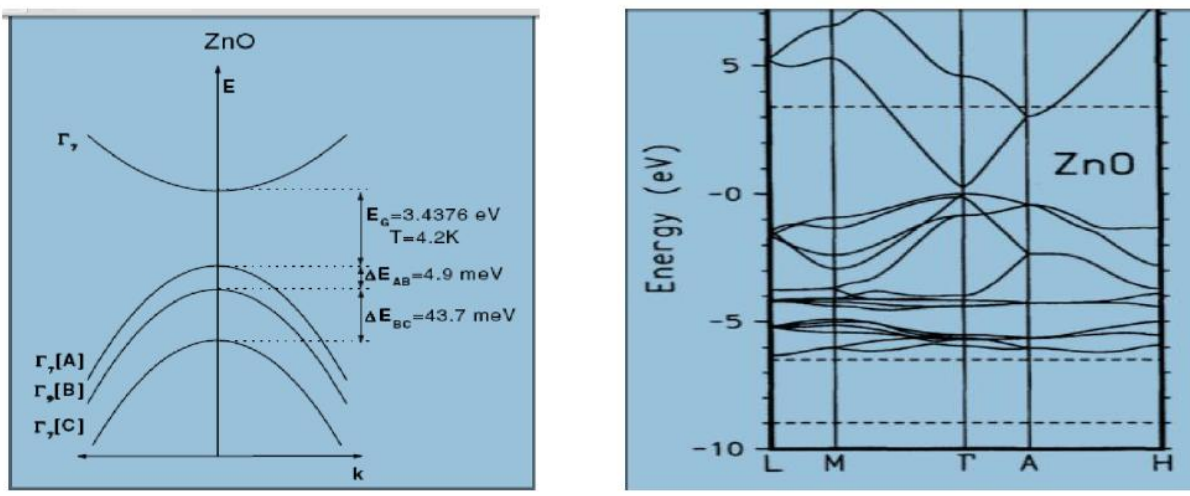

Figure.4: (a) Band structure and symmetries of hexagonal $\mathrm{ZnO}$. The splitting into three valence bands (A, B, C) is caused by crystal field and spin-orbit splitting.. (b) The LDA band structure of bulk wurtzite $\mathrm{ZnO}$ calculated using a standard pseudo potentials

\section{Application Of Zno Nanostructures}

Zinc oxide due to its versatility and multifunctionality creates attention in the research field related to its applications. A wide number of synthesis techniques also been developed by which $\mathrm{ZnO}$ can be grown in different nanoscale forms and thereby different novel nanostructures can be fabricated with different shapes ranging from nanowires to nanobelts and even nanosprings. Generally, nanobelts of $\mathrm{ZnO}$ can be obtained by sublimation of $\mathrm{ZnO}$ nanopowder without any catalyst. Each property of $\mathrm{ZnO}$ has its own applications. Starting from the wide band gap of $\mathrm{ZnO}$ makes it enable to form clusters consisting of $\mathrm{ZnO}$ nanocrystals and $\mathrm{ZnO}$ nanowires. Also due to the wide band gap, synthesis of $\mathrm{P}-\mathrm{N}$ homojunctions has been reported in some literatures but clarity on stability and reproducibility has not been established yet. Many fine optical devices can be fabricated based on the free-exciton binding energy in $\mathrm{ZnO}$ that is $60 \mathrm{MeV}$ because large exciton binding energy makes $\mathrm{ZnO}$ eligible to persist at room temperature and higher too. Since $\mathrm{ZnO}$ crystals and thin films exhibit second- and third-order non-linear optical behaviour, it can be used for non-linear optical devices. Thirdorder non-linear response has recently been observed in $\mathrm{ZnO}$ nano -crystalline films which make it suitable for integrated non-linear optical devices. Generally, the advantage of tuning the physical property of these oxides like zinc oxide becomes the root cause for the synthesis of smart application device. the electrical, optical, magnetic, and chemical properties can be very well tuned by making permutation and combination of the two basic structural characteristics they possess the is cations with mixed valence states, and anions with deficiencies(vacancies). Thus, making them suitable for several application fields such as semiconductor, superconductor, ferroelectrics, and magnetic. DSSCs is an optoelectronics device that converts light to electrical energy via charge separation in sensitizer dyes absorbed on a wide band gap semiconductor, which is different to conventional cells. One important difference between conventional and dry sensitized solar cell is that they are epitomized by silicon p-n junction solar cells. The demand for zinc oxide based dye-sensitized solar cell is due to its low fabrication cost.

\section{Characterisation Techniques}

In order to investigate various properties of the prepared sample, it has to goes under a number of characterisation techniques. The results of which gives the information about the different optical and structural properties of sample.

\subsection{Structural Characterisation}

In order to get exact information about the crystal structure, surface morphology, particle size etc. the following characterisation techniques are applicable.

1. XRD (X-ray Diffraction)

2. SEM (Scanning electron microscope)

3. Transmission electron microscope (TEM) 


\subsubsection{XRD (X-Ray Diffraction)}

Up to 1895 the study of matter at the atomic level was a difficult task but the discovery of electromagnetic radiation with $1 \AA(10-10 \mathrm{~m})$ wavelength, appearing at the region between gamma-rays and ultraviolet, makes it possible. As the atomic distance in matter is comparable with the wavelength of X-ray, the phenomenon of diffraction find its way through it and gives many promisable results related to the crystalline structure. The unit cell and lattices which are distributed in a regular three-dimensional way in space forms the base for diffraction pattern to occur. These lattices form a series of parallel planes with its own specific dspacing and with different orientations exist. The reflection of incident monochromatic X-ray from successive planes of crystal lattices when the difference between the planes is of complete number $\mathrm{n}$ of wavelengths leads to famous Bragg's law:

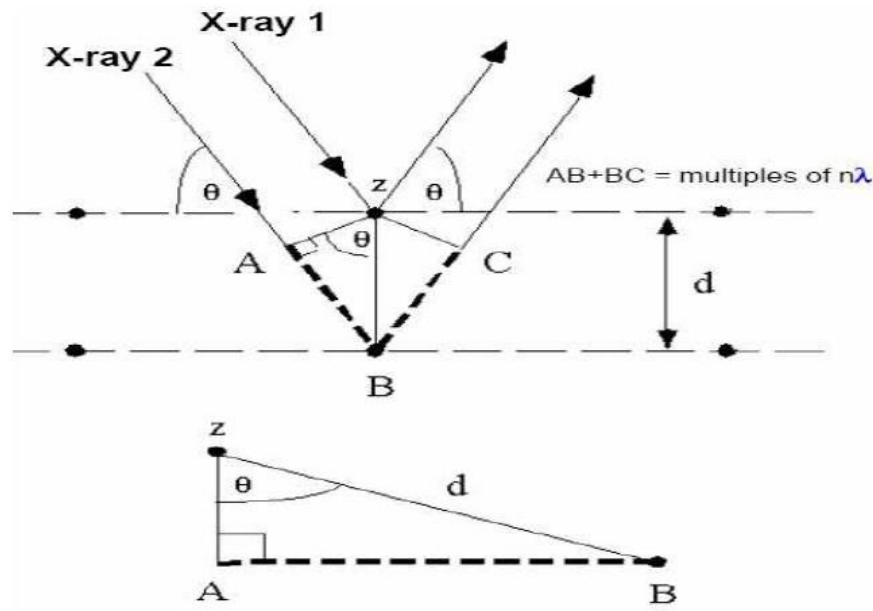

Figure 5: X-ray Diffraction in accordance with Bragg's Law

$$
\text { n } \lambda=2 \mathbf{d} \sin \theta
$$

Where $n$ is an integer $1,2,3 \ldots$. (Usually equal 1 ), $\lambda$ is wavelength in angstroms (1.54 $\AA$ for copper), $\mathrm{d}$ is interatomic spacing in angstroms, and $\theta$ is the diffraction angle in degrees. Plotting the angular positions and intensities of the resultant diffracted peaks of radiation produces a pattern, which is characteristic of the sample. The fingerprint characterization of crystalline materials and the determination of their structure are the two fields where XRD has been mostly used. Unique characteristic X-ray diffraction pattern of each crystalline solid gives the designation of "fingerprint technique" to XRD for its identification. XRD may be used to determine its structure, i.e. how the atoms pack together in the crystalline state and what the interatomic distance and angle are etc. From these points it can be concluded that X-ray diffraction has become a very important and powerful tool for the structural characterization in solid state physics and materials science.

\subsubsection{SEM (Scanning electron microscope)}

The scanning electron microscope (SEM) uses a focused beam of high-energy electrons to generate a variety of signals at the surface of solid specimens. The signals that derive from electron reveal information about the sample including external morphology (texture), chemical composition, and crystalline structure and orientation of materials making up the sample. In most applications, data are collected over a selected area of the surface of the sample, and a 2-dimensional image is generated that displays spatial variations in these properties. Areas ranging from approximately $1 \mathrm{~cm}$ to 5 microns in width can be imaged in a scanning mode using conventional SEM techniques (magnification ranging from $20 \mathrm{X}$ to approximately $30,000 \mathrm{X}$, spatial resolution of 50 to $100 \mathrm{~nm}$ ). The SEM is also capable of performing analyses of selected point locations on the sample; this approach is especially useful in qualitatively or semi-quantitatively determining chemical compositions (using EDS), crystalline structure, and crystal orientations (using EBSD)[50,53]. The design and function of the SEM is very similar to the EPMA and considerable overlap in capabilities exists between the two instruments. 


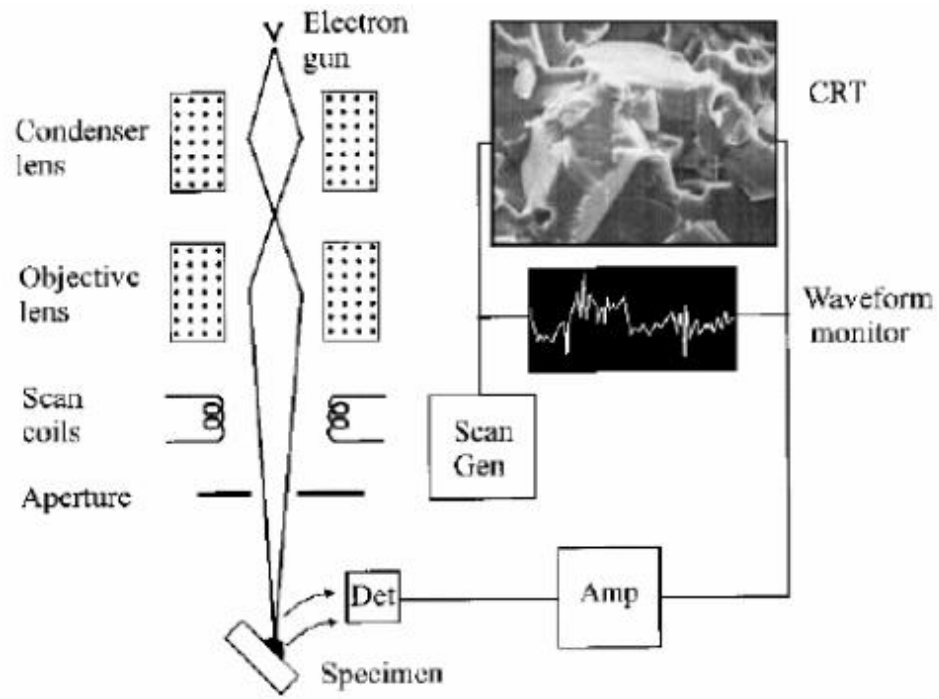

Figure 6: Schematic Diagram of SEM

Accelerated electrons in an SEM carry significant amounts of kinetic energy, and this energy is dissipated as a variety of signals produced by electron-sample interactions when the incident electrons are decelerated in the solid sample. These signals include secondary electrons (that produce SEM images), backscattered electrons (BSE), diffracted backscattered electrons (EBSD that are used to determine crystal structures and orientations of minerals), photons (characteristic X-rays that are used for elemental analysis and continuum X-rays), visible light (cathode luminescence--CL), and heat. Secondary electrons and backscattered electrons are commonly used for imaging samples: secondary electrons are most valuable for showing morphology and topography on samples and backscattered electrons are most valuable for illustrating contrasts in composition in multiphase samples (i.e. for rapid phase discrimination SEM analysis is considered to be "non-destructive"; that is, $x$-rays generated by electron interactions do not lead to volume loss of the sample, so it is possible to analyze the same materials repeatedly. The SEM is routinely used to generate high-resolution images of shapes of objects (SEI) and to show spatial variations in chemical compositions: 1) acquiring elemental maps or spot chemical analyses using EDS, 2)discrimination of phases based on mean atomic number (commonly related to relative density) using BSE, and 3) compositional maps based on differences in trace element "activators" (typically transition metal and Rare Earth elements) using CL. The SEM is also widely used to identify phases based on qualitative chemical analysis and/or crystalline structure. Precise measurement of very small features and objects down to $50 \mathrm{~nm}$ in size is also accomplished using the SEM.

\subsubsection{Transmission electron microscope (TEM)}

Electron Microscopes are scientific instruments that use a beam of highly energetic electrons to examine objects on a very fine scale. This examination can yield the information like topography, morphology, composition as well as crystallographic information's. Working principle is exactly as their optical counterparts except that they use a focused beam of electrons instead of light to "image" the specimen and gain information as to its structure and composition. The main use of this technique is to examine the specimen structure, composition or properties in sub microscopic details so that this microscopy technique is significantly involved in numerous fields. In TEM there is no change in the refractive index of the medium when the illumination beam is deflected, the vacuum in the lens is the same as the vacuum in the column. Deflection is in this case only due to the electromagnetic properties of the lens which are defined by electromagnetic plates that are only able to influence the path direction of the electrons, since all of the electrons carry a negative charge. Those electrons that pass through the sample go on to form the image while those that are stopped or deflected by dense atoms in the specimen are subtracted from the image. In this way a black and white image is formed. Remaining other electrons which passes close to heavy atom and get only slightly deflected make their way down the column and contribute to the image.. There are three main reasons why the microscope column must be operated under very high vacuum. The first of these is to avoid collisions between electrons of the beam and stray molecules. Such collisions can result in a spreading or diffusing of the beam or more seriously can result in volatization event if the molecule is organic in nature. Such volatizations can severely contaminate the microscope column especially in finely machined regions such as apertures and pole pieces that will serve to degrade the image. 


\subsection{Optical Characterisation}

As we know zinc oxide has wide range of application in the field of optoelectronics devices on putting the sample to following characterisation techniques gives information related to optical properties.

1. UV-Visible Spectroscopy

2. Fouriesr Transform Infrared Spectroscopy (IR).

\subsubsection{UV-Visible Spectroscopy}

The wavelength of UV is shorter than the visible light. It ranges from 100 to $400 \mathrm{~nm}$. In a standard UV$\mathrm{V}$ is spectrophotometer, a beam of light is split; one half of the beam (the sample beam) is directed through a transparent cell containing a solution of the compound being analysed, and one half (the reference beam) is directed through an identical cell that does not contain the compound but contains the solvent. The instrument is designed so that it can make a comparison of the intensities of the two beams as it scans over the desired region of the wavelengths. If the compound absorbs light at a particular wavelength, the intensity of the sample beam (IS) will be less than that of the reference beam. Absorption of radiation by a sample is measured at various wavelengths and plotted by a recorder to give the spectrum which is a plot of the wavelength of the entire region versus the absorption (A) of light at each wavelength. And the band gap of the sample can be obtained by plotting the graph between ( $\alpha \mathrm{h} v \mathrm{vs} \mathrm{h} v$ ) and extrapolating it along $\mathrm{x}$-axis. Ultraviolet and visible spectrometry is almost entirely used for quantitative analysis; that is, the estimation of the amount of a compound known to be present in the sample. The sample is usually examined in solution.

\subsubsection{Fourier Transform Infrared Spectroscopy (FTIS)}

In the region of longer wavelength or low frequency the identification of different types of chemicals is possible by this technique of infrared spectroscopy and the instrument requires for its execution is Fourier transform infrared (FTIR) spectrometer. The spectroscopy merely based on the fact that molecules absorb specific frequencies that are characteristic of their structure termed as resonant frequencies, i.e. the frequency of the absorbed radiation matches the frequency of the bond or group that vibrates. And the detection of energy is done on the basis of shape of the molecular potential energy surfaces, the masses of the atoms, and the associated vibronic coupling. Sometimes help of approximation techniques likeBorn-Oppenheimer and harmonic approximations are also taken. As each different material is a unique combination of atoms, no two compounds produce the exact same infrared spectrum. Therefore, infrared spectroscopy can result in a positive identification (qualitative analysis) of every different kind of material. In addition, the size of the peaks in the spectrum is a direct indication of the amount of material present.FTIR can be used to analyze a wide range of materials in bulk or thin films, liquids, solids, pastes, powders, fibers, and other forms. FTIR analysis can give not only qualitative (identification) analysis of materials, but with relevant standards, can be used for quantitative (amount) analysis. FTIR can be used to analyze samples up to $\sim 11$ millimeters in diameter, and either measure in bulk or the top $\sim 1$ micrometer layer.FTIR spectra of pure compounds are generally so unique that they are like a molecular "fingerprint".

\subsection{Thermal Analysis}

1. differential scanning calorimetry (DSC)

2. thermal gravimetric analysis (TGA)

\subsubsection{Differential Scanning Calorimetry (DSC)}

Thermal Analysis (STA) generally refers to the simultaneous application of Thermogravimetry (TGA) and Differential scanning calorimetry (DSC) to one and the same sample in a single instrument. The test conditions are perfectly identical for the TGA and DSC signals (same atmosphere, gas flow rate, vapour pressure of the sample, heating rate, thermal contact to the sample crucible and sensor, radiation effect, etc.).Thus, Thermal analysis (TA) is a group of techniques in which changes of physical or chemical properties of the sample are monitored against time or temperature, while the temperature of the sample is programmed which may involve heating or cooling at a fixed rate, holding the temperature constant (isothermal), or any sequence of these.

\subsubsection{Working of DSC}

The sample and reference chambers are heated equally into a temperature regime in which a transformation takes place within the sample. As the sample temperature deviates from the reference temperature, the device detects it and reduces the heat input to one cell while adding heat to the other, so as to maintain a zero temperature difference between the sample and reference. The quantity of electrical energy per unit time which must be supplied to the heating element in order to maintain null balance is assumed to be proportional to the heat released per unit time by the sample. 


\subsubsection{Working of TGA}

Measurements of changes in sample mass with temperature can made using a thermobalance. This is a combination of a suitable electronic microbalance with a furnace and associated temperature programmer. The balance should be in an enclosed system so that the atmosphere can be controlled. $\mathrm{Y}$ axis is \%mass loss; $\mathrm{X}$ axis is temp (or time, since usually a linear heating rate).

As the specimen changes weight, its tendency to rise or fall is detected by LVDT. A current through the coil on the counterbalance side exerts a force on the magnetic core which acts to return the balance pan to a null position. The current required to maintain this position is considered proportional to the mass change of the specimen

\section{Conclusion}

The structural, optical and electronic properties of $\mathrm{ZnO}$ nanoparticles were investigated. Nanostructured materials can have significantly different properties, depending on the chosen fabrication route. Each method offers some advantages over other techniques while suffering limitation from the others.

At present, there are many methods to investigate various properties of the prepared sample, in which a number of characterisation techniques are being used. Their results provide the information about the different structural, optical and electronics properties of the sample. We can characterise the crystals structure and pure phase by using XRD diffraction patterns. The optical properties of the samples are investigated by measuring the UV-VIS absorption at room temperature. It is found that with increasing calcinations temperature the band gap of the samples remains almost same.

\section{Acknowledgment}

Authors are grateful to Dr. Satyam Pincha and Mr. Avinash Pareek for providing the needed help.

\section{References:}

[1] A.R. Hutson, Phys.Rev.,108(1957)222-230.

[2] G.Heiland,E.Mollwo,F.Stockmann, Solid State Phys.,8(1959)193-196

[3] J.R.Haynes, Phys.Rev.Lett.,17(1966)16.

[4] Y.S.Park et al, Phys.Rev.,143(1966)2.

[5] T.Damen,S.P.S Porto,B.Tell, Phys.Rev.,142(1966)2

[6] M.Matsuoka,J.J. App.Phys.,10(1971)736.

[7] P.R. Emtage,J. App.Phys.,48(1977)4372-4384

[8] M.Inada,J.J. Phys.,17(1978)1-10.

[9] F.S.Hickerne,J. App.Phys.,44(1973),1061-1071.

[10] Z.L. Wang, J. Phys. Condensed Matter, 16(2004)829.

[11] K. H. Hellwege, O. Madelung, A. M. Hellege,19(1987)119-595.

[12] Z. L. Wang, J.Phys., Condens. Matter, 16(2004)829-858.

[13] G.H. Lee, Y. Yamamoto , M. Kourogia, M. Ohtsua, Thin Solid Films, 386(2001)117 -120.

[14] B. K. Meyer, H. Alves, D. M. Hofmann, W. Kriegseis et al., Phys. Stat. Sol.,241(2004)231-260.

[15] TingtingRen, Holly R. Baker, Kristin M. Poduska, Thin Solid Films, 515(2007)7976-7983.

[16] PijusKanti Samanta and Partha Roy Chaudhuri, ScienceofAdvanced Mater.,3(2011)107-112.

[17] Chang Shi Lao et al., Nanoletters,6(2006)263-266.

[18] S. K. Mohanta, D. C. Kim, B. H. Kong, H. K. Cho, W. Liu, and S. Tripathy, Sci. Adv. Mater.,2(2010)64.

[19] R Ding, J. Liu, J. Jiang, X. Ji, X. Li, F. Wu, and X. Huang, Sci. Adv.Mater.,2(2010)396.

[20] Z. L. Wang, Mater. Sci. Eng.33(2009)64.

[21] Y. Ding and Z. L. Wang, Micron.,40(2009)335.

[22] M. Wei1, D. Zhi, and J. L. MacManus-Driscoll, Nanotechnology, 16(2005)1364.

[23] J. Jie, G. Wang, X. Han, Q. Yu, Y. Liao, G. Li, and J. G. Hou, Chem.Phys. Lett. 387(2004),466.

[24] J. Zhang, W. Yu, and L. Zhang, Phys. Lett. A 299(2002)276.

[25] M. H. Huang, S. Mao, H. Feick, H. Yan, Y. Wu, H. Kind, E. Weber,R. Russo, and P. Yang, Science,292(2001)1897.

[26] P. X. Gao, Y. Ding, and Z. L. Wang, Nano Lett., 9(2009)137.

[27] B. Tang, H. Deng, Z. W. Shui, and Q. Zhang, J. Nanosci. Nanotechnol.10(2010)1842.

[28] M. Vafaee, M. SasaniGhamsarib,*,Materials Letters, 61 (2007)3265-3268.

[29] M. Veith, M. Haas, and V. Huch, Chem. Mater.,17(2005)95-101.

[30] Kang X.Ya, Wang TianDiao, Han Yin, Tao MinDe, Mater. Research Bulletin,32(1997),1165-1171.

[31] Eric A. Meulenkamp,J. Phys. Chem. B.,102(1998)5566-5572.

[32] M. Risti et al., Journal of Alloys and Compounds ,397 (2005)L1-L4. 